\title{
Resolving spectral overlap issue of intermediate band solar cells using non-uniform sub-bandgap state filling
}

\author{
Albert Lin $^{1 *}$ and Jamie Phillips ${ }^{2}$ \\ 1 Institute of Electronics Engineering, National Chiao-Tung University, Hsinchu, Taiwan \\ 2 Department of Electrical Engineering and Computer Science, University of Michigan, Ann Arbor, MI, USA
}

\begin{abstract}
Intermediate band solar cell provides novel alternative to multi-junction solar cell, but its efficiency is significantly degraded when spectral overlap exists between different absorption bands. Here, a scheme using non-uniform sub-bandgap state filling together with intermediate band transport is proposed to resolve the spectral overlap issue. On the basis of detailed balance calculation, spectrally decoupled devices using low-high state filling is shown to achieve $52.8 \%$ conversion efficiency when $4 \mathrm{eV}$ spectral overlap is present between absorption coefficients of different bands, compared with baseline efficiency equal to $35.1 \%$ for conventional half-filled intermediate band devices. If a base material without intermediate band is added to the two section low-high state filling devices, the efficiency is further increased to $61.5 \%$, which approaches efficiency of $63.2 \%$ for intermediate band devices with no spectral overlap and $63.8 \%$ for unconstrained triple-junction tandem cells. The junction thermalization loss associated with proposed new structures is shown to be equal to conventional half-filled intermediate band devices. Copyright @ 2013 John Wiley \& Sons, Ltd.
\end{abstract}

\section{KEYWORDS}

solar cell; intermediate state/band; spectral overlap; sub-bandgap photovoltaics

* Correspondence

Albert Lin, Institute of Electronics Engineering. National Chiao-Tung University, Hsinchu, Taiwan.

E-mail: hdtd5746@gmail.com

Received 4 August 2012; Revised 9 November 2012; Accepted 3 December 2012

\section{INTRODUCTION}

Spectral overlap between different absorption processes is a fundamental problem for solar cells based on multi-photon processes, often referred to as intermediate band solar cells (IBSC) [1-20]. Ideally, the incident solar photons are absorbed by the appropriate highest threshold process to minimize thermalization of photoexcited carriers. In this approach, two sub-bandgap photons excite an electron from the valence band (VB) to conduction band $(\mathrm{CB})$ via intermediate levels and contribute to one electron-hole pair. In common semiconductor materials, there is a high density of states beyond the band edge, enabling higher energy photons to participate in lower energy optical transitions leading to undesired energy loss via carrier thermalization. The resulting spectral overlap between optical transitions can span an energy range of several electron volts, where the efficiency of the solar cell can drop to a value below a standard solar cell without intermediate electron states [17]. The use of intermediate band absorption coefficient that is an order of magnitude smaller than the valence to conduction band absorption has been proposed to approach ideal efficiency
[4], but this may lead to thicker absorber thickness for full absorption[17]. In this study, the influence of varied occupation of intermediate electron states is examined as a means of spatially separating sub-bandgap optical transitions. This approach is shown in Figure 1(b), where the intentional low-high (LH) occupation provides a means of preventing optical transitions into/out of the intermediate states. This approach relies on charge carrier transport via the intermediate band to transfer electrons between spatially separated absorption bands. The conversion efficiency of these structures is examined as a function of spectral overlap and compared with conventional IBSC structures and triple-junction solar cells. The initial result of this work can be found in [21]

\section{EQUIVALENCE OF JUNCTION THERMALIZATION LOSS}

There are mainly two different thermalization loss mechanisms in solar cells. First, high energy photons excite hot electrons. The other is the junction thermalization loss 


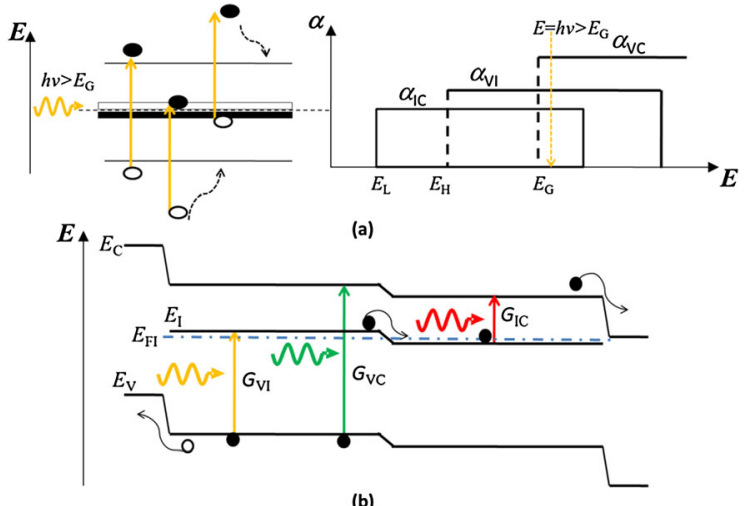

Figure 1. (a) Illustration of spectral overlap and associated thermalization (left figure, dashed arrows). (b) Illustration of entire device structure of low-high state filling cells.

where carriers laterally transport over a potential drop and thus lose excess energy. The first thermalization loss is why IBSC or multi-junction cell is so important, and the second thermalization loss is the motivation for solar concentrators. Figure 2 illustrates the equivalence of junction thermalization loss for conventional IBSC structure and LH state filling IBSC structure. The photons of different energy excite two sub-bandgap transitions. The photo-generated carrier must go through lateral-transport processes in order to be collected by p-emitter and n-emitter. The junction thermalization loss associated with lateraltransport includes $\mathrm{VB}$ to p-emitter $\Delta E_{\mathrm{V}, \mathrm{PL}}, \mathrm{CB}$ to n-emitter $\Delta E_{\mathrm{C}, \mathrm{HN}}$, and from low to high state filling region in IB $\Delta E_{\mathrm{I}, \mathrm{LH}}$. For conventional IBSC, the total junction thermalization (TJT) loss is

$$
\begin{aligned}
\Delta E_{T J T} & =\Delta E_{\mathrm{V}, \mathrm{PL}}+\Delta E_{\mathrm{C}, \mathrm{HN}}=\Delta E_{\mathrm{V}, \mathrm{PL}}+\Delta E_{\mathrm{V}, \mathrm{HN}} \\
& =V_{\mathrm{bi}}-V_{\mathrm{a}}
\end{aligned}
$$

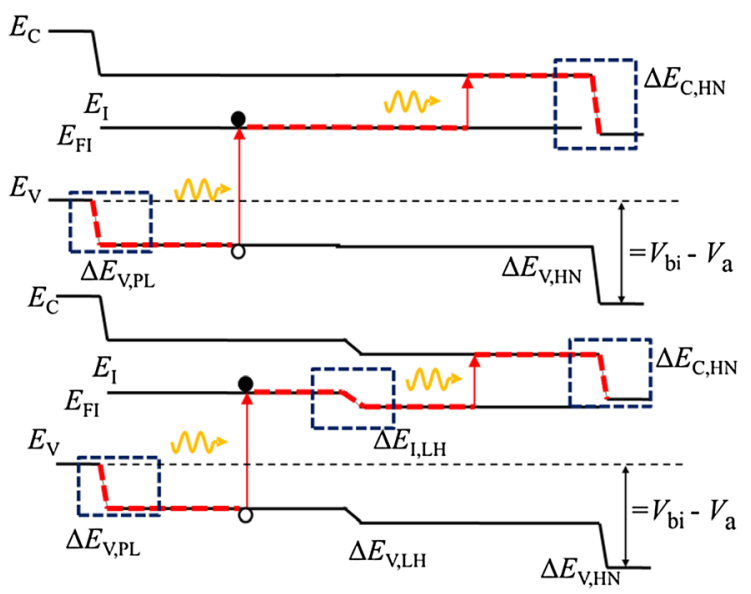

Figure 2. Illustration of photo-generated carrier transport routes. Comparison of baseline intermediate band solar cell (IBSC) and low-high state filling IBSC. where $V_{\mathrm{bi}}$ is built-in potential and $V_{\mathrm{a}}$ is applied voltage. For LH state filling IBSC, the TJT loss is

$$
\begin{aligned}
\Delta E_{\mathrm{TJT}} & =\Delta E_{\mathrm{V}, \mathrm{PL}}+\Delta E_{\mathrm{I}, \mathrm{LH}}+\Delta E_{\mathrm{C}, \mathrm{HN}} \\
& =\Delta E_{\mathrm{V}, \mathrm{PL}}+\Delta E_{\mathrm{V}, \mathrm{LH}}+\Delta E_{\mathrm{V}, \mathrm{HN}} \\
& =V_{\mathrm{bi}}-V_{\mathrm{a}}
\end{aligned}
$$

Thus, the TJT loss is the same for LH state filling structure and conventional half-filled (HF) structure.

\section{SOLAR CELL MODEL}

The detailed balance approach is a self-consistent thermodynamic argument that provides an estimate on the highest efficiency that can be achieved by photovoltaic solar cells. This study follows the prior models and associated assumptions $[2,4,10,11,13-15,17]$, with the modification of a spatially dependent optical absorption coefficient based on intermediate band occupation for analysis in one dimension. Spatially dependent generation and recombination processes are then evaluated to determine integrated values of current density and corresponding conversion efficiency.

The derivation for solar cell efficiency based on intermediate band electron state occupation starts from the photon number, which may be represented by

$$
\begin{gathered}
\frac{\mathrm{d} v}{\mathrm{~d} x}=-\alpha_{\mathrm{VC}}\left(v-v_{\mathrm{CV}}\right)-\alpha_{\mathrm{IC}}\left(v-v_{\mathrm{CI}}\right)-\alpha_{\mathrm{VI}}\left(v-v_{\mathrm{IV}}\right) \\
\\
\alpha_{\mathrm{VC}}=\alpha_{\mathrm{VC} 0} \\
\alpha_{\mathrm{IC}}=\alpha_{\mathrm{IC} 0} \zeta \\
\alpha_{\mathrm{VI}}=\alpha_{\mathrm{VI} 0}(1-\zeta)
\end{gathered}
$$

where $\alpha_{\mathrm{VC}}, \alpha_{\mathrm{IC}}$, and $\alpha_{\mathrm{VI}}$ are the respective absorption coefficient for VB to $\mathrm{CB}$, IB to $\mathrm{CB}$ and VB to IB transitions, respectively, $\zeta$ is the Asbeck factor characterizing intermediate state occupation. The assumption of the "HF" condition in the original derivation $[4,10,11,14,15,17]$ corresponds to $\zeta=0.5$ in this analysis. The values of $v_{\mathrm{XY}}(\mathrm{X}, \mathrm{Y}=\mathrm{C}, \mathrm{I}$, or $\mathrm{V}$ ) are given by

$$
v_{\mathrm{XY}}=\frac{1}{1+\exp \left(\frac{E_{\mathrm{XY}}-\mu_{\mathrm{XY}}}{k T}\right)}
$$

where $E$ is energy, $k$ is Boltzman constant, and $\mu_{\mathrm{XY}}=$ $E_{\mathrm{FX}}-E_{\mathrm{FY}}$ is the separation between quasi-Fermi levels. It should be noted that $\mu_{\mathrm{CV}}=E_{\mathrm{FC}}-E_{\mathrm{FV}}=V_{\mathrm{a}}$ is the applied bias to the diode.

The photon rate equation given in Equation (3) holds for the entire base region, which may be solved piecewise for the case of spatially varying absorption coefficient. The photon number at a particular section $\mathrm{i}$ in the base region can be expressed as follows: 


$$
\begin{aligned}
v_{\mathrm{i}}(x)= & \frac{\alpha_{\mathrm{VC}, \mathrm{i}} v_{\mathrm{CV}}+\alpha_{\mathrm{IC}, \mathrm{i}} v_{\mathrm{CI}}+\alpha_{\mathrm{VI}, \mathrm{i}} v_{\mathrm{IV}}}{\alpha_{\mathrm{VC}, \mathrm{i}}+\alpha_{\mathrm{IC}, \mathrm{i}}+\alpha_{\mathrm{VI}, \mathrm{i}}} \\
& \times\left[1-\exp ^{-\left(\alpha_{\mathrm{VC}, \mathrm{i}}+\alpha_{\mathrm{IC}, \mathrm{i}}+\alpha_{\mathrm{VI}, \mathrm{i}}\right) \times\left(x-x_{0}\right)}\right] \\
& +v_{\mathrm{i}}\left(x_{0}\right) \exp ^{-\left(\alpha_{\mathrm{VC}, \mathrm{i}}+\alpha_{\mathrm{IC}, \mathrm{i}}+\alpha_{\mathrm{VI}, \mathrm{i}}\right) \times\left(x-x_{0}\right)}
\end{aligned}
$$

where $x_{0}$ is the boundary of the particular piecewise section of the base region. $\alpha_{\mathrm{VC}, \mathrm{i}}, \alpha_{\mathrm{VC}, \mathrm{i}}, \alpha_{\mathrm{VC}, \mathrm{i}}$ is the absorption coefficient at section $i$, depending on the state filling of that section. Thus, the photon number can be written as

$$
v(x)= \begin{cases}v_{1}(x) & \text { for } \quad 0 \leq x \leq W_{1} \\ v_{2}(x) & \text { for } \quad W_{1} \leq x \leq W_{1}+W_{2}\end{cases}
$$

where $W_{1}$ and $W_{2}$ are the widths of the first and second sections, respectively. The net electron and hole generationrecombination rate can be calculated by integration $[4,10,15,17]$

$$
\begin{aligned}
(G-R)_{\mathrm{e}, \mathrm{i}}(x)= & \int_{E, \Omega} \alpha_{\mathrm{VC}, \mathrm{i}}\left(v-v_{\mathrm{CV}}\right) \\
& +\alpha_{\mathrm{IC}, \mathrm{i}}\left(v-v_{\mathrm{CI}}\right) \frac{2 E^{2}}{h^{3} c^{2}} \mathrm{~d} E \mathrm{~d} \Omega \\
(G-R)_{\mathrm{h}, \mathrm{i}}(x)= & \int_{E, \Omega} \alpha_{\mathrm{VC}, \mathrm{i}}\left(v-v_{\mathrm{CV}}\right) \\
& +\alpha_{\mathrm{VI}, \mathrm{i}}\left(v-v_{\mathrm{IV}}\right) \frac{2 E^{2}}{h^{3} c^{2}} \mathrm{~d} E \mathrm{~d} \Omega
\end{aligned}
$$

where $h=6.626 \times 10^{-34} \mathrm{~J} \mathrm{~s}$ is Plank's constant, $c=3 \times 10^{8}$ $\mathrm{m} / \mathrm{s}$ is the speed of light, and $\mathrm{d} \Omega=\sin \theta \mathrm{d} \theta \mathrm{d} \phi$ is the solid angle, $\theta$ is zenith angle, $\phi$ is azimuth angle, and sun is at positive $z$-axis direction. Substituting Equation (7) into Equation (8) and arranging terms, there will be three terms corresponding to the contribution of external generation, external recombination, and internal recombination, and the detailed balance together with photon recycling is accurately described. The details can be referred to $[10,11,17]$. The total electron or hole current density due to generation-recombination (dominant mechanism for ideal case) can be calculated by integrating over the total basewidth $W$

$$
\begin{aligned}
J_{e, h}= & \int_{0^{1}}^{W_{1}}(G(x)-R(x))_{\mathrm{e} / \mathrm{h}, 1} \mathrm{~d} x \\
& +\int_{W_{1}+W_{2}}^{W_{1}}(G(x)-R(x))_{\mathrm{e} / \mathrm{h}, 2} \mathrm{~d} x
\end{aligned}
$$

The current density depends on the quasi-Fermi level separation $\mu_{\mathrm{CI}}, \mu_{\mathrm{IV}}$, and $\mu_{\mathrm{CV}} . \mu_{\mathrm{CI}}$ and $\mu_{\mathrm{IV}}$ can be solved by knowing $\mu_{\mathrm{CI}}+\mu_{\mathrm{IV}}=\mu_{\mathrm{CV}}=V_{\mathrm{a}}$ and the requirement that electron and hole current density must be balanced in order to maintain charge neutrality and particle conservation in the entire base region. The current density is then solved for a given applied bias $V_{\mathrm{a}}$, where the efficiency is defined as

$$
\eta=\frac{J_{\mathrm{sc}} \times V_{\mathrm{oc}} \times F F}{P_{\mathrm{sun}}}
$$

where $J_{\mathrm{sc}}$ is the short-circuit current density, $V_{\mathrm{oc}}$ is the opencircuit voltage, $F F=J_{\mathrm{m}} V_{\mathrm{m}} / J_{\mathrm{sc}} V_{\mathrm{oc}}$ is the fill factor, $J_{\mathrm{m}}$ and $V_{\mathrm{m}}$ are the current density and voltage at the operating point where the power output from the solar cell is maximum, and $P_{\text {sun }}$ is the incident solar power density.

In this study, the bandgap energy and intermediate band position are assigned values of $E_{\mathrm{G}}=1.95 \mathrm{eV}$ and $E_{\mathrm{I}}=1.24$ $\mathrm{eV}$, respectively, based on the optimal values obtained from A. Luque's original detailed balance calculations $[17,20]$. Calculations are performed with solar concentration at either $X=1$ (one sun) or 46050 (fully concentrated sunlight) as indicated in figure captions. Values for absorption coefficients $\alpha_{\mathrm{VC}}, \alpha_{\mathrm{IC}}, \alpha_{\mathrm{VI}}$ are chosen to be $10^{4} \mathrm{~cm}^{-1}$ at the ideal HF IB condition, as representative values reported in previously published work $[4,8,10,15,17]$.

Four device structures are studied as shown in Figure 3 to compare the influence of spatially varying occupation of intermediate band states on spectral overlap and solar cell conversion efficiency. The first two device structures are (A) uniform absorber with HF IB and (B) absorber with nearly empty IB in half of the device and nearly filled in the other half of the device. It is assumed that efficient

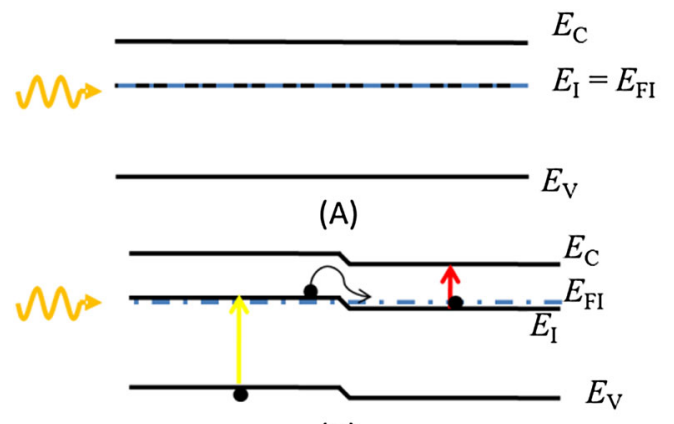

(B)

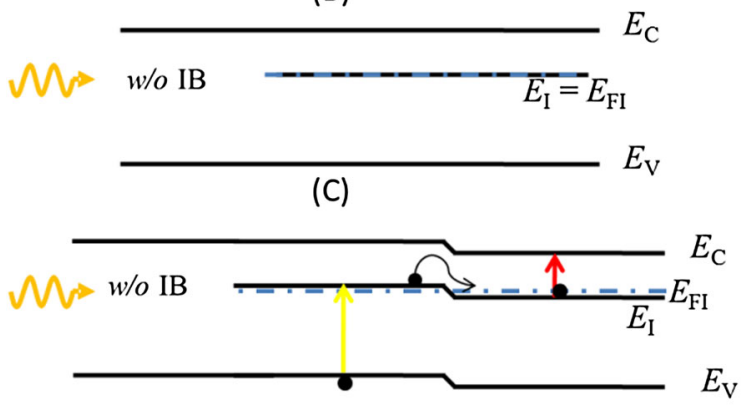

(D)

Figure 3. (A) Half-filled (HF), (B) spectrally decoupled (SD) device using low-high state filling, (C) HF + base semiconductor, (D) full SD. 
carrier transports in the IB between the two regions. Structure (A) serves as a point of reference, whereas structure (B) is the primary structure under study where a base region with empty IB in one region prevents IB to $\mathrm{CB}$ transitions and filled in one region prevents $\mathrm{VB}$ to $\mathrm{IB}$ transitions. Structures (C) and (D) are essentially the same as (A) and (B), respectively, but each contains an additional section without IB states to further spatially separate absorption processes. The regions without IB states are beneficial in ensuring solar photons with energy $E>E_{\mathrm{G}}$ are absorbed by the VB to $\mathrm{CB}$ transition instead of other low-threshold processes such as the VB to IB transition. (B) is referred to as spectrally decoupled (SD) devices and (D) is referred to as full SD devices. It should be noted that the proposed spatially varying occupation of intermediate band states may be realized in principle by doping and control over impurity/nanostructure placement, providing spectral separation of absorption bands while maintaining a single-junction device structure.

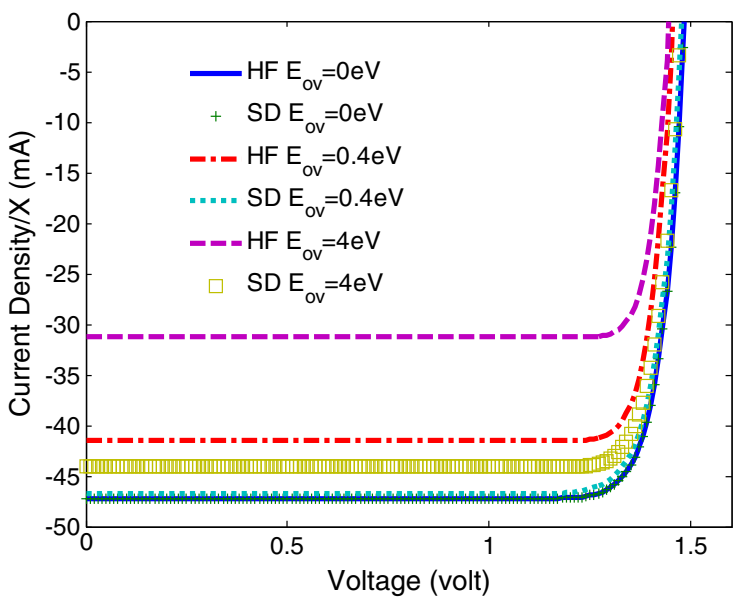

Figure 4. $J-V$ for spectrally decoupled cells. $\left(\zeta_{1}, \zeta_{2}\right)=(0.1,0.9)$. $W=2 \mu \mathrm{m} . X=1$.

\section{RESULTS}

The current-voltage characteristics of the device structures are shown in Figure 4. The $J_{\mathrm{sc}}$ and $V_{\mathrm{oc}}$ for the device with HF IB demonstrates a clear decrease with spectral overlap $E_{\mathrm{ov}}$, whereas the decrease in $J_{\mathrm{sc}}$ and $V_{\mathrm{oc}}$ is significantly reduced for the structure with separate LH IB occupation regions. For LH state filled devices, photons with energy $E_{\mathrm{H}}<E<E_{\mathrm{G}}$ are absorbed in the first section of the device via the VB to IB transition, whereas photons with energy $E_{\mathrm{L}}<E<E_{\mathrm{H}}$ are not absorbed because of the low density of available electrons in the IB available for absorption. This configuration ensures that photons are absorbed by the highest threshold process. Photo-excited electrons may then transport to the second section of the base region to be subsequently excited to the $\mathrm{CB}$ by solar photons with energy $E_{\mathrm{L}}<E<E_{\mathrm{H}}$. For the HF IB device B, all photons with energy $E>E_{\mathrm{L}}$ can be absorbed by the IB to $\mathrm{CB}$ transition where excess electron energy of $E-E_{\mathrm{L}}$ is lost by phonon emission. Without spectral overlap $\left(E_{\mathrm{ov}}=0\right)$, the intermediate band devices behave identically, where distinctions become pronounced with increasing spectral overlap.

The influence of the spatially varying absorption for $E_{\mathrm{ov}}=0 \mathrm{eV}$ is illustrated in Figure 5, where sub-bandgap absorption occurs selectively according to electron state occupation.

Increasing spectral overlap results in more pronounced differences in absorption, as shown in Figure 6. One clear characteristic is observed in Figure 6(a), where there is significant re-emission for the $\mathrm{CB}$ to $\mathrm{IB}$ transition. The re-emission is due to the spectral overlap, where high energy photons are absorbed by inappropriate low-threshold processes $\left(E_{\mathrm{L}}<E<E_{\mathrm{H}}\right)$, leading to significant thermalization. The thermalized high-energy photons then recombine through low threshold CB to IB transitions, assumed to be radiative in this analysis. The recombination rate in the context of detailed balance theory is proportional to exp $(-E / k T)$ and thus the low-threshold $\mathrm{CB}$ to IB transition $\left(E_{\mathrm{L}}<E<E_{\mathrm{H}}\right)$ is the most efficient recombination process.
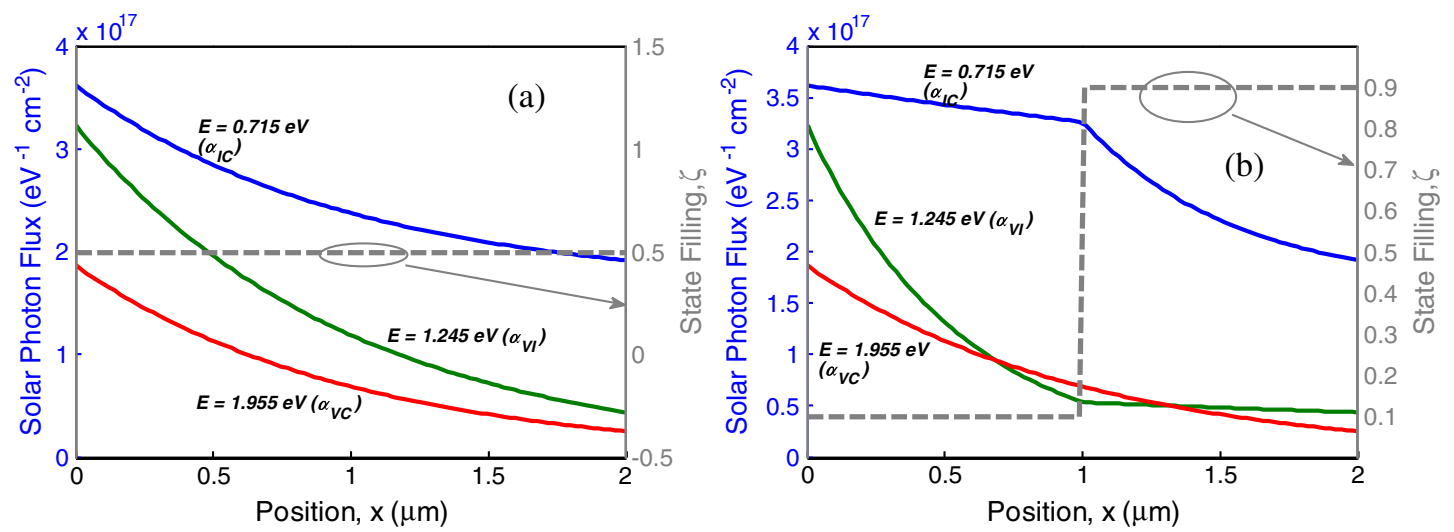

Figure 5. Spectral photon absorption profile for $E_{\mathrm{ov}}=0 \mathrm{eV}, X=1$, at short-circuit condition. (a) An HF device, (b) an SD device with $\left(\zeta_{1}, \zeta_{2}\right)=(0.1,0.9)$. The photon energy is $0.005 \mathrm{eV}$ above the transition threshold. 

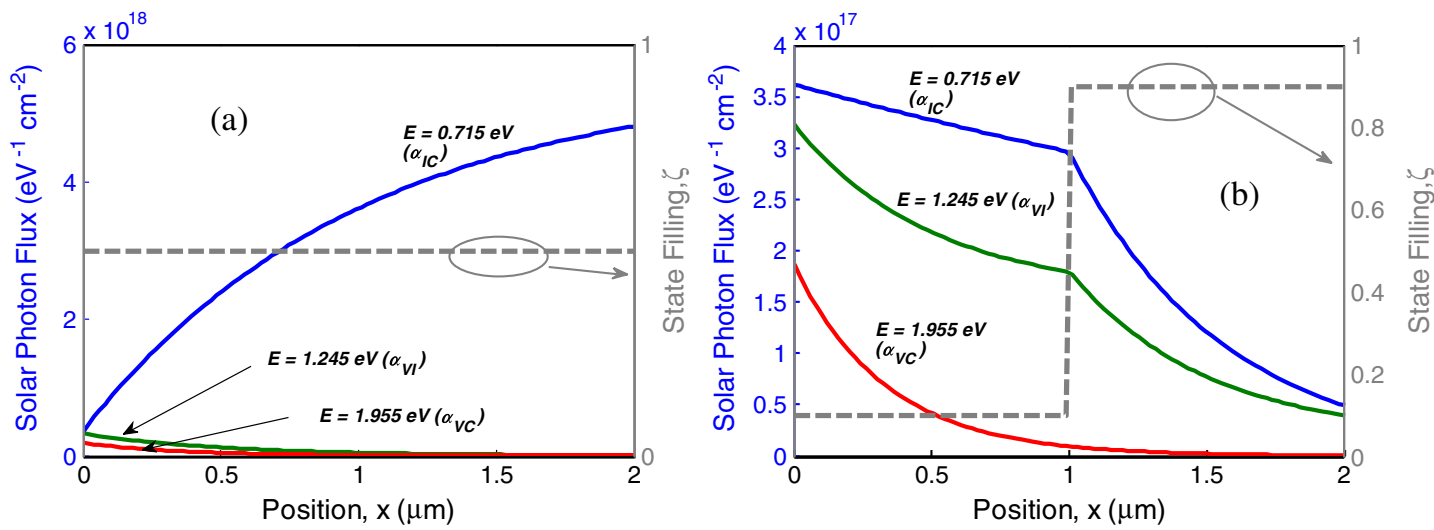

Figure 6. Spectral photon absorption profile $E_{\mathrm{ov}}=4 \mathrm{eV}, X=1$ at short-circuit condition. (a) An HF device, (b) an SD device with $\left(\zeta_{1}, \zeta_{2}\right)=(0.1,0.9)$. The photon energy is $0.005 \mathrm{eV}$ above the transition threshold.

This is consistent with experimental observation where thermal generation recombination is more severe in small bandgap materials. Notice this does not mean that total number of photon increases with distance, because the photon number is plotted at single energy at $0.005 \mathrm{eV}$ above $\alpha_{\text {IC }}$ transition threshold to show the effect of strong recombination through $\alpha_{\mathrm{IC}}$. For photon energy several $k T$ away from $\alpha_{\mathrm{IC}}$ transition threshold, photon number still decreases with distance. In the case of the two-section structure (B) (Figure 3), the re-emission is suppressed since LH IB occupation decouples the spectral overlap between $\alpha_{\mathrm{IC}}$ and $\alpha_{\mathrm{VI}}$. The recombination through efficient $\alpha_{\text {IC }}$ process can still exist, because there is still spectral overlap and parasitic absorption in the case of $\left(\zeta_{1}, \zeta_{2}\right)=(0.1,0.9)$, but this effect is not very pronounced unless the energy very close to $\alpha_{\mathrm{IC}}$ transition threshold is plotted. The efficiency of structure (B) is therefore much closer to the ideal $E_{\mathrm{ov}}=0$ condition even if spectral overlap is present.

The dependence of conversion efficiency on spectral overlap $\left(E_{\mathrm{ov}}\right)$ more clearly illustrates the impact of spatially varying IB population, as shown in Figure 7. For no

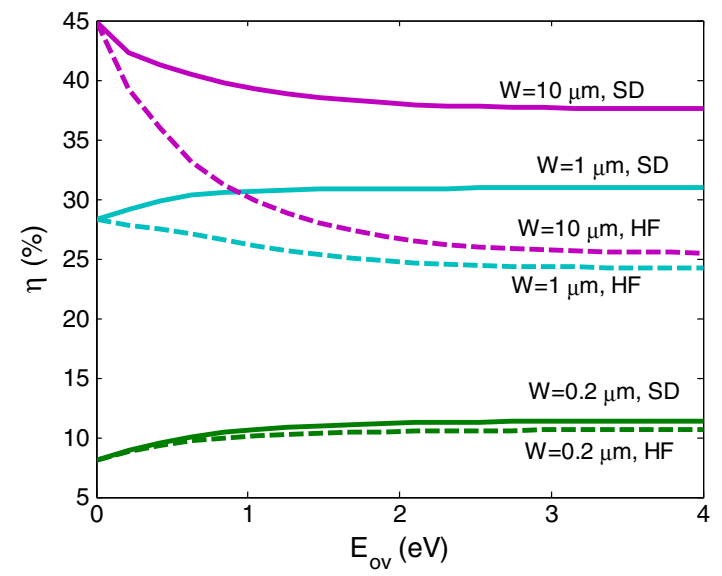

Figure 7. $\eta$ vs. $E_{\mathrm{ov}}$ for varying $W_{\text {tot. }}\left(\zeta_{1}, \zeta_{2}\right)=(0.1,0.9) . X=1$. spectral overlap $\left(E_{\mathrm{ov}}=0 \mathrm{eV}\right)$, conversion efficiency is the same for all IBSC device structures studied because solar photons are absorbed by the highest threshold process. Device structures with an HF IB show a rapid decrease with increasing $E_{\mathrm{ov}}$ due to thermalization of photo-generated electrons or holes in the $\mathrm{CB}$ and $\mathrm{VB}$ for optical transitions excited that are not the highest possible energy transitions, similar to calculations reported in previously published work [17]. An anomaly in the figure is that the efficiency of both $\mathrm{HF}$ and SD devices increases with $E_{\mathrm{ov}}$ for short basewidth due to more absorption resulting from spectral overlap. The efficiency for device structures with variable intermediate band occupation shows a significantly lower sensitivity to spectral overlap and higher efficiency compared with their HF counterparts.

The dependence of efficiency on the occupation level of the IB states is clearly an important factor, as shown in Figure 8 for LH state filling SD devices. In the analysis, IB occupation is assumed to be symmetric in the two regions such that $\zeta_{2}=1-\zeta_{1}$. It is clear that the contrast in occupation for the two regions $\left(\zeta_{1}, \zeta_{2}\right)=(0.1,0.9)$ is sufficient to achieve high efficiency, where only small

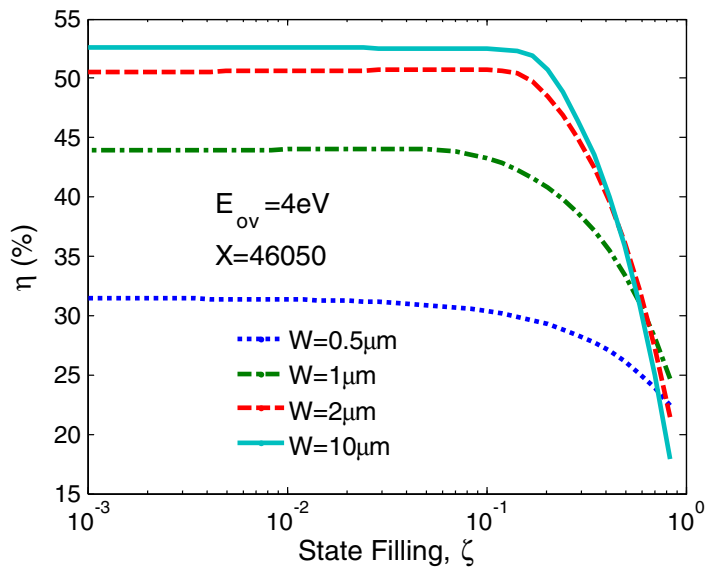

Figure 8. $\eta$ vs. $\zeta$. for varying $W, E_{\mathrm{ov}}=4 \mathrm{eV} . X=46050$ 
improvements are achieved beyond these values. High occupation in the first section of the base region $\left(\zeta_{1}>0.5\right)$ corresponds to high absorption of the low energy transition nearest the incident surface, resulting in a decrease in efficiency to below the value for the case of a half-occupied IB due to increased thermalization losses. This behavior is similar to the requirement for multi-junction cells where incidence at the large bandgap side is essential.

The dependence of conversion efficiency on base width is shown in Figure 9 for the various device structures (Refer to Figure 3). The full spectrally decoupled device (D) is essential to further increasing the efficiency toward the $E_{\mathrm{ov}}=0$ efficiency limit to eliminate the spectral overlap still present between $\alpha_{\mathrm{IC}}$ (or $\alpha_{\mathrm{VI}}$ ) and $\alpha_{\mathrm{VC}}$. In the structure (D), the three regions of the device completely separate/ decouple the three absorption bands to minimize thermalization losses. The highest efficiencies calculated in Figure 9 are $35.1 \%$ for structure (A), $45.0 \%$ for structure (B), $52.83 \%$ for structure (C), and $61.5 \%$ for structure (D) (refer to Figure 3). It should be noted that the efficiency is calculated to be $63.2 \%$ for the ideal IBSC [11], $63.8 \%$ for the unconstrained triple-junction tandem cell [22], and $63.2 \%$ for the series constrained triple-junction tandem cell [23]. The fully decoupled structure (D) provides

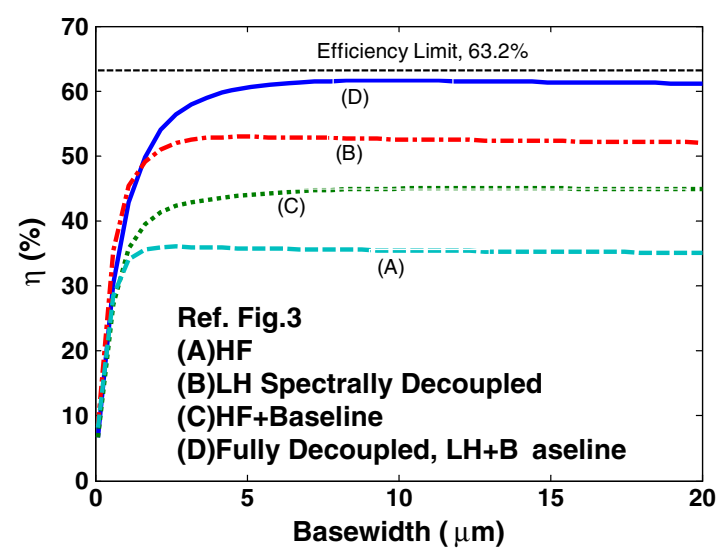

Figure 9. $\eta$ vs. W. for varying scheme (refer to Figure 3 ). $\left(\zeta_{1}, \zeta_{2}\right)=(0.01,0.99)$ for spectrally decoupled (SD) and full SD scheme. $E_{\mathrm{ov}}=4 \mathrm{eV} . X=46050$.

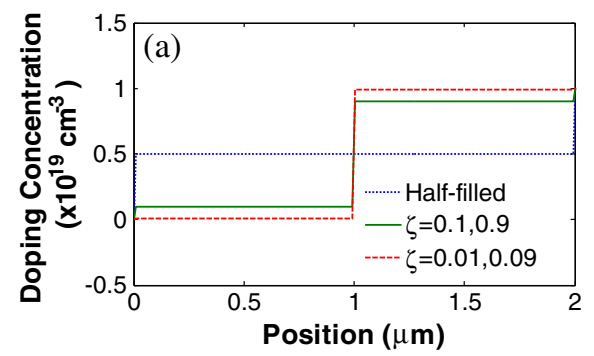

a theoretical efficiency limit $>60 \%$ that approaches the efficiency limit of both the ideal IBSC and triple-junction solar cell. The SD structure essentially provides a 'triple-band' structure analogous to a triple-junction solar cell, while maintaining a single-junction device.

\section{PRACTICAL IMPLEMENTATION OF LOW-HIGH STATE FILLING}

The absorber layer structure with non-uniform intermediate band occupation may be implemented in practice by variable doping levels. The electrostatic potential $(\psi)$ is affected by several different charge contributions:

$$
\vec{\nabla} \cdot \varepsilon_{r} \varepsilon_{0} \vec{\nabla} \psi=-q\left(N_{D}^{+}-N_{A}^{-}-n+p-\zeta N_{I}\right)
$$

The Poisson equation for electrostatic potential $(\psi)$ includes the charge density for electrons $(n)$, holes $(p)$, ionized acceptors $\left(N_{\mathrm{A}}^{-}\right)$, ionized donors $\left(N_{\mathrm{D}}^{+}\right)$, and charge in the intermediate band represented by the occupation of states $(\zeta)$ and concentration of intermediate band states $\left(N_{\mathrm{I}}\right), \varepsilon_{\mathrm{r}}$ is relative permittivity and $\varepsilon_{0}=8.84 \times 10^{-12} \mathrm{~F} / \mathrm{m}$ is the permittivity in vacuum. In this calculation, $N_{\mathrm{I}}=10^{19} \mathrm{~cm}^{-3}, \varepsilon_{\mathrm{r}}=10$, basewidth $W=2 \mu \mathrm{m}$, and the width for $p$-and $n$-contact layers are $0.3 \mu \mathrm{m}$ with heavy doping concentration of $10^{19} \mathrm{~cm}^{-3}$. The IB is assumed to lie at a fixed energy level in the bandgap. Alternative density of states functions with IB states distributed in energy may be similarly defined. The doping profile and resulting occupation of IB states are shown in Figure 10, where the n-type doping profile in the base region is varied for three cases of $0.5 N_{\mathrm{I}}(\mathrm{HF}),\left(0.1 N_{\mathrm{I}}\right.$, $\left.0.9 N_{\mathrm{I}}\right)$, and $\left(0.01 N_{\mathrm{I}}, 0.99 N_{\mathrm{I}}\right)$. The resulting energy band diagram is shown in Figure 11, where only small variation in potential energy results in large variation in IB occupation as a result of the exponential dependence of occupation on potential according to Fermi-Dirac statistics. The energy band profile is very similar to IB devices with HF IB where flat band region exists in the base region, implying a minimal effect of variable IB occupation of these device structures on carrier transport in the base region.

Figure 10. (a) Doping versus position, (b) $\zeta$ versus position. 

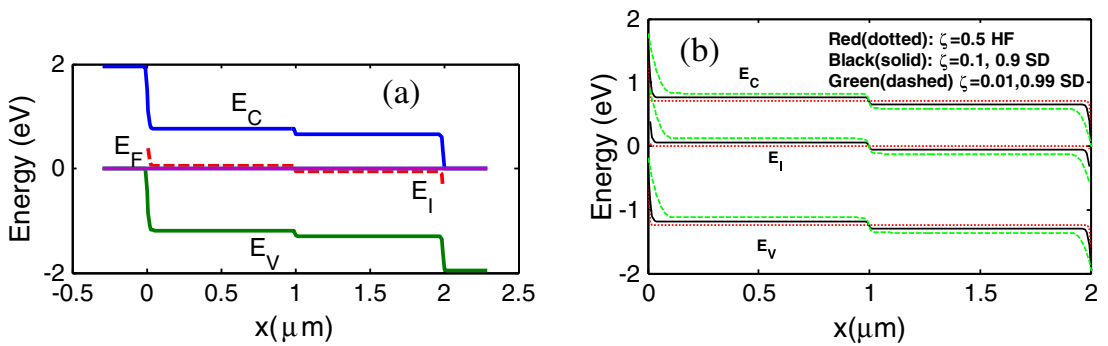

Figure 11. (a) Band diagram of entire device, (b) band diagram for varying $\zeta$ at base region.

\section{CONCLUSION}

The efficiency of a device spatially decoupling VB-IB and IB-CB absorption by defining a non-uniform occupation of IB states is calculated to be $52.8 \%$ for the case of significant spectral overlap between bands. This efficiency is significantly higher than the efficiency of $35.1 \%$ calculated for devices with IB states at uniform $50 \%$ occupation. A full spectral decoupling scheme for $\mathrm{CB}-\mathrm{VB}, \mathrm{VB}-\mathrm{IB}$, and IB-CB transitions may further increase the conversion efficiency, where a value of $61.5 \%$ is calculated for the case of $4 \mathrm{eV}$ spectral overlap, approaching the detailed balance efficiency limit of $63.2 \%$ for IBSC devices with no spectral overlap. The success of this approach is reliant on the ability to spatially vary IB occupation by doping or other mechanisms, and to realize efficient transport via the IB. These two challenges are identified as key areas of future experimental research to enable high-efficiency IBSC devices.

\section{ACKNOWLEDGEMENTS}

The initial IBSC device modeling principles were done at the University of Michigan supported as part of the Center for Solar and Thermal Energy Conversion, an Energy Frontier Research Center funded by the U.S. Department of Energy, Office of Science, Office of Basic Energy Sciences under Award Number DE-SC0000957. Detailed studies of the high-low state filling principles were done at National Chiao-Tung University, funded by the National Science Council (NSC), Taiwan and supported by high speed computing facilities from National Center of HighPerformance Computing (NCHC), Hsinchu, Taiwan.

\section{REFERENCES}

1. Aroutiounian V, Petrosyan S, Khachatryan A, Touryan K. Quantum dot solar cells. Journal of Applied Physics 2001; 89: 2268. DOI: 10.1063/1.1339210

2. Brown AS, Green MA. Intermediate band solar cell with many bands: ideal performance. Journal of Applied Physics 2003; 94, 6150-6158. DOI: 10.1063/ 1.1610774
3. Cress CD, Hubbard SM, Landi BJ, Raffaellea RP, Wilt DM. Quantum dot solar cell tolerance to alpha-particle irradiation. Applied Physics Letters 2007, 91: 183108. DOI: $10.1063 / 1.2803854$

4. Cuadra L, Martí A, Luque A. Influence of the overlap between the absorption coefficients on the efficiency of the intermediate band solar cell. IEEE Transactions on Electron Devices 2004; 51: 1002. DOI: 10.1109/ TED.2004.828161

5. Yu KM, Walukiewicz W, Ager JW, Bour D, Farshchi R, Dubon OD, Li SX, Sharp ID, Haller EE. Multiband GaNAsP quaternary alloys. Applied Physics Letters 2006; 88: 092110-1-092110-3. DOI: 10.1063/1.2181627

6. Laghumavarapu RB, Moscho A, Khoshakhlagh A, El-Emawy M, Lester LF, Huffakerb DL. GaSb/GaAs type II quantum dot solar cells for enhanced infrared spectral response. Applied Physics Letters 2007; 90: 173125. DOI: $10.1063 / 1.2734492$

7. Leya M, Boudaden J, Kuznicki ZT. Thermodynamic efficiency of an intermediate band photovoltaic cell with low threshold Auger generation, Journal of Applied Physics, 2005 vol. 98, pp. 044905-1-044905-5. DOI: $10.1063 / 1.2010622$

8. Lin AS, Wang W, Phillips JD. Model for intermediate band solar cells incorporating carrier transport and recombination and application to $\mathrm{ZnTeO}$. Journal of Applied Physics 2009; 105: 064512. DOI: 10.1063/ 1.3093962

9. Luque A. Operation of the intermediate band solar cell under non-ideal space charge region conditions and half filling of the intermediate band. Journal of Applied Physics 2006; 99: 094503. DOI: 10.1063/ 1.2193063

10. Luque A, Marti A. A metallic intermediate band high efficiency solar cell. Progress in Photovoltaics: Research and Applications 2001; 9: 73. DOI: 10.1002/ pip.354

11. Luque A, Mart1 A. Increasing the efficiency of ideal solar cells by photon induced transitions at intermediate levels. Physical Review Letters 1997; 78: 5014. DOI: 10.1103/PhysRevLett.78.5014 
12. Luque A, Martí A, Cuadra L. Impact-ionizationassisted intermediate band solar cell. IEEE Transactions on Electron Devices 2003; 50: 447-454. DOI: 10.1109/TED.2003.809024

13. Luque A, Martí A, Stanley C, López N, Cuadra L, Zhou D, Pearson JL, McKee A. General equivalent circuit for intermediate band devices: potentials, currents and electroluminescence. Journal of Applied Physics 2004; 96: 903-909. DOI: 10.1063/1.1760836

14. Luque A, Martí A, Cuadra L. Thermodynamic consistency of Sub-bandgap absorbing solar cell proposals. IEEE Transactions on Electron Devices 2001; 49: 2118-2124. DOI: 10.1109/16.944204

15. Martí A, Cuadra L, Luque A. Quasi-drift diffusion model for the quantum dot intermediate band solar cell. IEEE Transactions on Electron Devices 2002; 49: 1632. DOI: 10.1109/TED.2002.802642

16. Marti A, Lopez N, Antolín E, Canovas E, Luque A, Stanley CR, Farmer CD, Diaz P. Emitter degradation in quantum dot intermediate band solar cells. Applied Physics Letters 2007; 90: 233510. DOI: 10.1063/1.2747195

17. Navruz TS, Saritas M. Efficiency variation of the intermediate band solar cell due to the overlap between absorption coefficients. Solar Energy Materials \& Solar Cells 2008; 92: 273. DOI: 10.1016/j.solmat.2007.08.012
18. Stanley CR, Intermediate-band solar cells and the quantum dot approach. Department of Electronics and Electrical Engineering, University of Glasgow, Glasgow, G12 8LT, UK2004.

19. G Wei, SR Forrest. Intermediate-band solar cells employing quantum dots embedded in an energy fence barrier. Nano Letters 2007; 7: 218-222. DOI: 10.1021/ n1062564s

20. Wei G, Shiu K-T, Giebink NC, Forrest SR. Thermodynamic limits of quantum photovoltaic cell efficiency. Applied Physics Letters 2007; 91: 223507. DOI: 10.1063/1.2817753

21. Lin A, Phillips J. Decoupling spectral overlap of intermediate band solar cells using low-high state filling. In IEEE Photovoltaic Specialist Conference, Austin: USA, 2012.

22. Vos AD. Detailed balance limit of the efficiency of tandem solar cells. Journal of Physics D: Applied Physics 1980; 13: 839-846. DOI: 10.1088/00223727/13/5/018

23. Brown AS, Green MA. Detailed balance limit for the series constrained two terminal tandem solar cell. Physica E: Low-dimensional Systems and Nanostructures. 2002; 14: 96-100. DOI: 10.1016/S1386-9477 (02)00364-8 